\title{
Function of $\mathrm{Ca}^{2+}$-/calmodulin-dependent protein kinase $\mathrm{IV}$ in $\mathrm{Ca}^{2+}$-stimulated neuronal signaling and behavior
}

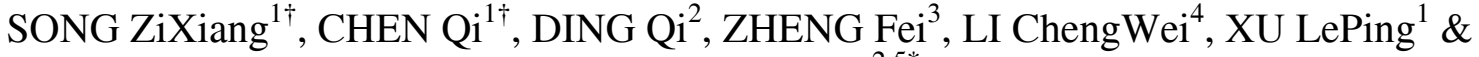 \\ WANG HongBing ${ }^{2,5^{*}}$
}

\author{
${ }^{1}$ Department of Adolescent Psychiatry and Behavioral Therapy, Heping Hospital, Changzhou 213003, China; \\ ${ }^{2}$ Department of Physiology, Michigan State University, East Lansing, MI 48824, USA; \\ ${ }^{3}$ Department of Biochemistry and Molecular Biology, Michigan State University, East Lansing, MI 48824, USA; \\ ${ }^{4}$ College of Life Science and Agronomy, Zhoukou Normal University, Zhoukou 466001, China; \\ ${ }^{5}$ Neuroscience Program, Michigan State University, East Lansing, MI 48824, USA
}

Received September 26, 2014; accepted October 16, 2014

\begin{abstract}
The activity of $\mathrm{Ca}^{2+} /$ calmodulin-dependent protein kinase IV (CaMKIV) is sensitive to activity-dependent changes in the level of intracellular $\mathrm{Ca}^{2+}$. Following neuronal stimulation, the activation of CaMKIV may trigger synaptic modifications and transcriptional responses, both of which are involved in regulating cognitive and emotional behavior. Here, we used CaMKIV knockout (KO) neurons and mice to examine the function of CaMKIV in $\mathrm{Ca}^{2+}$-stimulated intracellular signaling and animal behavior, respectively. Following NMDA receptor activation or membrane depolarization, the up-regulation of CREB (cAMP responsive element binding protein) and its target gene $B d n f$ (brain-derived neurotrophic factor) was intact in cortical neurons obtained from CaMKIV KO mice. CaMKIV KO mice displayed severe impairment in contextual fear memory but normal locomotor activity and anxiety level in the contextual training chamber. Although CaMKIV KO mice showed normal memory in the standard passive avoidance task, they were defective in learning the temporal dissociative passive avoidance task. As indicated by the light/dark test and marble-burying test data, CaMKIV KO mice showed less anxiety and normal perseveration. In the voluntary wheel-running test, CaMKIV KO mice showed normal running time and distance but higher maximal running speed. Our results demonstrate the function of CaMKIV in regulating different forms of fear memory, anxiety, and certain aspect of motor function.
\end{abstract}

animal behavior, calmodulin, CREB, ERK1/2, intracellular signaling, learning and memory

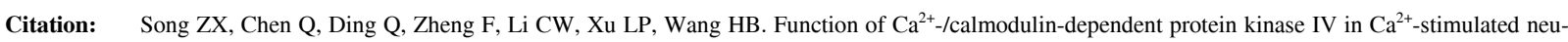
ronal signaling and behavior. Sci China Life Sci, 2015, 58: 6-13, doi: 10.1007/s11427-014-4781-4

$\mathrm{Ca}^{2+}$ is a major intracellular small messenger molecule that regulates many aspects of neuronal function. Transient rise in intracellular $\mathrm{Ca}^{2+}$ through ion channel-mediated influx or release from the internal storage triggers signaling cascade and, in turn, impinges on dynamic synaptic and nuclear responses. Aberrant $\mathrm{Ca}^{2+}$ homeostasis and $\mathrm{Ca}^{2+}$-stimulated

$†$ Contributed equally to this work

*Corresponding author (email: wangho@msu.edu) intracellular signaling are often associated with neurological dysfunction or diseases [1,2]. As a major $\mathrm{Ca}^{2+}$ effector molecule, calmodulin ( $\mathrm{CaM}$ ) senses the changes in intracellular $\mathrm{Ca}^{2+}$ and tunes the activity of numerous protein kinases such as CaMKs [3]. CaMKIV is expressed in distinct brain regions that regulate learning and memory, emotion, and motor function. Although CaMKIV has been demonstrated to phosphorylate synaptic proteins, its function is mainly implicated in regulating activity-dependent nuclear responses 
such as gene transcription [3,4].

Previous studies have demonstrated that the transcription factor CREB and its co-activator CBP (CREB binding protein) are regulated by the serine-threonine kinase activity of CaMKIV [5-7]. The current understanding emphasizes that the CaMKIV-dependent phosphorylation of CREB at serine 133 (Ser133) enhances its transcription activity. The transcription of CREB-regulated genes has been shown to be up-regulated following neuronal activity (e.g., c-Fos transcription) [8] and learning (e.g., Bdnf transcription) [9]. In brain slices and cortical neurons, glutamate- and membrane depolarization (by $\mathrm{KCl}$ )-stimulated CREB phosphorylation are ablated in CAMKIV mutant samples [10]. The up-regulation of CREB phosphorylation in vivo is also absent in the hippocampus of CaMKIV knockout (KO) mice following physical restraint [10] or fear conditioning [11]. However, it is not clear whether CaMKIV regulates the basal level of CREB phosphorylation in non-stimulated neurons.

Consistent with the notion that the CREB-mediated transcription is critical for neuroplasticity and adaptive behaviors, loss of activity-dependent CREB phosphorylation in CaMKIV KO mice correlates with the impairment in long-term potentiation (LTP) [10] and defective fear memory [10]. CaMKIV KO mice also display less anxiety-related behavior and stress response [12]. However, a recent study used an independent line of CaMKIV KO mice and found inconsistent phenotypes [13]. Interestingly, while spatial memory is un-affected in CaMKIV KO animals, it is significantly impaired in CREB mutants [14]. This implies that CaMKIV and CREB may differentially regulate the activity-dependent genetic response, and CREB activity may not be solely controlled by the CaMKIV-mediated Ser133 phosphorylation.

In this study, we examined the function of CaMKIV in CREB phosphorylation and $B d n f$ transcription following the activation of the NMDA receptors (NMDAR) and the L-type voltage-gated calcium channels (L-VGCC). We also performed an array of behavioral test with CaMKIV KO mice. We identified new functions of CaMKIV in passive avoidance memory and motor activity. Our data also help to consolidate some inconsistent results from the previous studies.

\section{Materials and methods}

\subsection{Animals}

CaMKIV KO mice on C57BL6 background were obtained from the Jackson Laboratories, and maintained in the University Laboratory Animal Resources facility at Michigan State University. Animals had free access to water and food, and were kept under the 12-h light/12-h dark housing condition. Two- to four-month-old male mice from different litters were pooled together, and subjected to behavioral anal- ysis. Separate cohorts were used for different behavioral tests. All protocols were approved by the Institutional Animal Care and Use Committee at Michigan State University.

\subsection{Primary neuronal culture and stimulation}

Cortices were obtained from CaMKIV $\mathrm{KO}$ and wild type (WT) mice on postnatal day 0 . The preparation and maintenance of the primary cortical culture were performed as described in our previous study [15]. DIV 8 (days in vitro 8) neurons were stimulated with $50 \mathrm{mmol} \mathrm{L}^{-1} \mathrm{KCl}$ along with

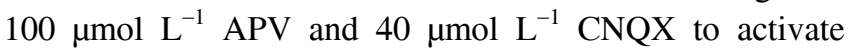
L-VGCC. Incubation with $15 \mu \mathrm{mol} \mathrm{L}{ }^{-1} \mathrm{NMDA}$ and $2 \mu \mathrm{mol}$ $\mathrm{L}^{-1}$ glycine along with $10 \mu \mathrm{mol} \mathrm{L}{ }^{-1}$ nifedipine and $40 \mu \mathrm{mol}$ $\mathrm{L}^{-1}$ CNQX was used to activate NMDAR.

\subsection{Western blot and analysis of $B d n f$ transcription}

For Western blot analysis, samples were harvested 15, 30, or $60 \mathrm{~min}$ following neuronal stimulation. Equal amount of total protein from different samples was separated by SDS polyacrylamide gel electrophoresis, and transferred to nitrocellulose membrane. The levels of CREB phosphorylation at Ser133 (pCREB) and $\beta$-actin were detected by specific antibodies (1:1,000 dilution for pCREB antibody, Upstate, Lake Placid, NY, USA; 1:5,000 for $\beta$-actin antibody, Sigma, St Louise, MO, USA). The immuno-signal was obtained by using the Odyssey system (LI-COR Biosciences, USA), and quantified by using the Scion Image software (Scion Corp. Frederick, Maryland, USA).

The level of exon I- and exon-IV containing as well as the level of total $B d n f$ mRNA was determined by semi-quantitative RT-PCR as described in our previous studies $[15,16]$. Total RNA was purified from samples collected $1 \mathrm{~h}$ after neuronal stimulation.

\subsection{Behavioral analysis}

All behavioral tests were performed between ZT4 and ZT8. For contextual fear conditioning (CFC), mouse was introduced to the training chamber and allowed to freely explore for $1 \mathrm{~min}$ before the delivery of a mild foot shock $(0.7 \mathrm{~mA}$, $2 \mathrm{~s}$ ). The mouse remained in the chamber for $30 \mathrm{~s}$ after the shock, and was returned to its home cage. The trained animals were placed into the same chamber $24 \mathrm{~h}$ later, and the fear response was determined by the percentage of freezing (immobility) during the 2-min test. During both training and testing, the ambulatory travel distance and movement time were determined by the TruScan activity system (Coulbourn Instruments, USA).

To determine the locomotor open field activity in the CFC training chamber, new cohorts of mice were placed in the chamber for $15 \mathrm{~min}$. Their activities including ambulatory distance, total distance traveled in the margin and cen- 
ter areas, and numbers of entries to the center area of the arena were determined by the TruScan activity system.

For passive avoidance, the apparatus is a box that consists of a lit chamber and a dark chamber. These two chambers are separated by a wall equipped with a trap door. During training, mouse was introduced to the lit half of the training chamber, and allowed to explore for $1 \mathrm{~min}$ before the trap door opened. Immediately after the animal entered the dark chamber, a mild electric foot shock $(0.7 \mathrm{~mA}$ with $2 \mathrm{~s}$ duration) was delivered. The animal remained in the dark chamber for $30 \mathrm{~s}$ after the shock, and was then returned to its home cage. The animals were tested $24 \mathrm{~h}$ later. The crossover latency for animals to enter the dark chamber from the lit chamber was recorded.

For temporal dissociative passive avoidance (TDPA), mouse was introduced to the lit chamber, and allowed to explore for $1 \mathrm{~min}$ before the trap door was lifted. When animal entered the dark chamber, the trap door was dropped. A mild foot-shock $(0.7 \mathrm{~mA}$ for $2 \mathrm{~s})$ was delivered $5 \mathrm{~min}$ after animal entered the dark chamber. The animal remained in the dark chamber for $20 \mathrm{~s}$ after the foot shock, and then was returned to its home cage. The TDPA training was performed daily for $3 \mathrm{~d}$. The final testing was on day 4 . The time animal spent in the lit chamber (i.e., between the time at trap door lifting and the time at animal entering the dark chamber) was recorded as crossover latency.

During the light/dark test, mouse was introduced to the dark chamber, and habituated for $2 \mathrm{~min}$ before the entry door to the lit chamber was lifted open. The mouse was allowed to move freely between the dark and lit chamber for $10 \mathrm{~min}$. The latency of animals' initial crossover to the lit chamber was recorded. The total time spent in the lit chamber and the number of entry to the lit chamber were also recorded.

The marble-burying test was considered as a paradigm to measure repetitive behavior (perseveration) [17]. The animal was first habituated in a standard mouse box filled with $5 \mathrm{~cm}$-thick bedding for $1.5 \mathrm{~h}$. Then, 15 marbles $(1 \mathrm{~cm}$ diameter) were placed evenly in the box (five rows with three marbles in each row). Thirty minutes later, the number of buried marbles was counted.

To determine the activity of wheel-running, animal was singly housed in a large mouse box equipped with a running wheel. The mouse was habituated for $7 \mathrm{~d}$ with the wheel being locked. After un-locking the wheel, the running activities including running distance and time as well as maximal speed were recorded for $7 \mathrm{~d}$ with a bicycle computer/ odometer (CATEYE ${ }^{\circledR}$ VELO 9).

\subsection{Statistic analysis}

All data are presented as mean \pm SEM. Two-way ANOVA or repeated measures two-way ANOVA or Student's $t$-test was used to determine statistic difference, which was considered when $P<0.05$ was reached.

\section{Results}

2.1 Genetic deletion of CaMKIV does not affect CREB phosphorylation and $B d n f$ transcription following the activation of NMDAR and L-VGCC

Previous studies have suggested that CaMKIV is required for the activity-dependent phosphorylation of CREB at Ser133 [10,11], leading to transcriptional up-regulation of CREB target genes. In cultured cortical neurons, we did not find difference in the basal level of pCREB in nonstimulated WT and CaMKIV KO neurons. Following the stimulation with NMDA, the level of pCREB was significantly increased in both WT and CaMKIV KO neurons (Figure 1A). Among the CREB target genes, Bdnf has been shown to be regulated by CREB both in vivo and in vitro $[15,18,19]$. Here, we found that the total Bdnf mRNA level, before and after NMDA stimulation, was comparable between WT and CaMKIV KO neurons (Figure 1B). Because the consensus CRE was identified in promoter I and IV of the $B d n f$ gene [16], we further determined the level of exon I- and exon IV-containing Bdnf mRNAs. Deletion of CaMKIV also had no significant effect (Figure 1B).

Another important source contributing to $\mathrm{Ca}^{2+}$-stimulated CREB activation is the L-VGCC-mediated $\mathrm{Ca}^{2+}$ influx [20]. We activated L-VGCC by KCl-induced membrane depolarization. We found comparable up-regulation of pCREB (Figure 1C) and Bdnf (including total, exon I-, and exon IV-containing mRNA) transcription (Figure 1D) in WT and CaMKIV KO neurons.

\subsection{Genetic deletion of CaMKIV impairs contextual fear memory without affecting shock sensitivity and lo- comotion}

During contextual training, the mild electric foot shock elicited similar increase of locomotor movement in WT and CaMKIV KO mice (Figure 2A), indicating that both groups of animals had similar sensory response to the aversive shock. Locomotor movement decreased to a similar level immediately after the foot hock in both groups, indicating comparable learning in WT and CaMKIV KO mice (Figure $2 \mathrm{~A}$ and B). However, when tested $24 \mathrm{~h}$ later, CaMKIV KO animals showed significantly less freezing (Figure 2C) and more movement (Figure 2D) than WT mice. These data demonstrate that CaMKIV is required for contextual fear memory retention.

One possibility that CaMKIV KO mice showed less freezing may be due to alterations in locomotor activity or intrinsic anxiety level. Thus, we determined these measures in the same contextual training chamber. During the 15-min test, new cohorts of WT and CaMKIV KO mice displayed similar movement (Figure $3 \mathrm{~A}$ and $\mathrm{B}$ ). There was also no significant difference in their activity in the center (Figure $3 \mathrm{~B}$ and $\mathrm{C}$ ) and margin areas (Figure 3B) of the chamber. 
A

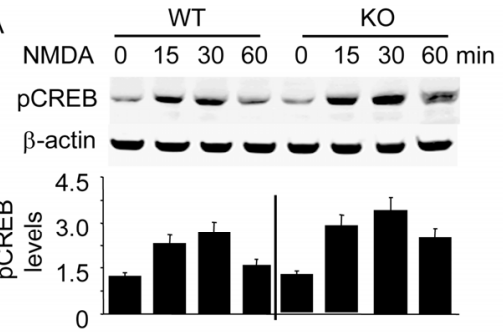

B
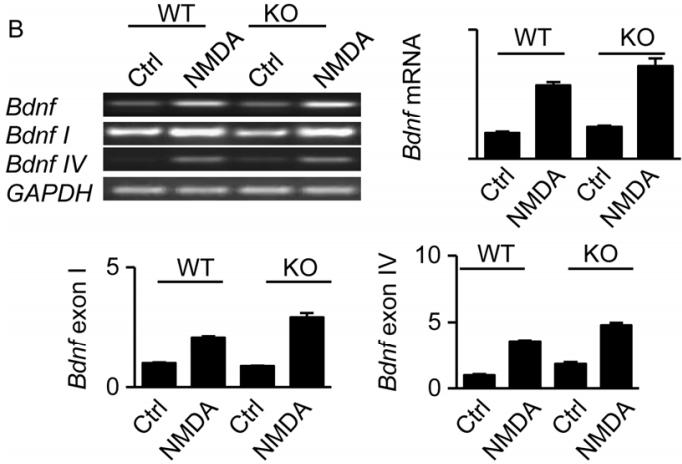

$\mathrm{C}$
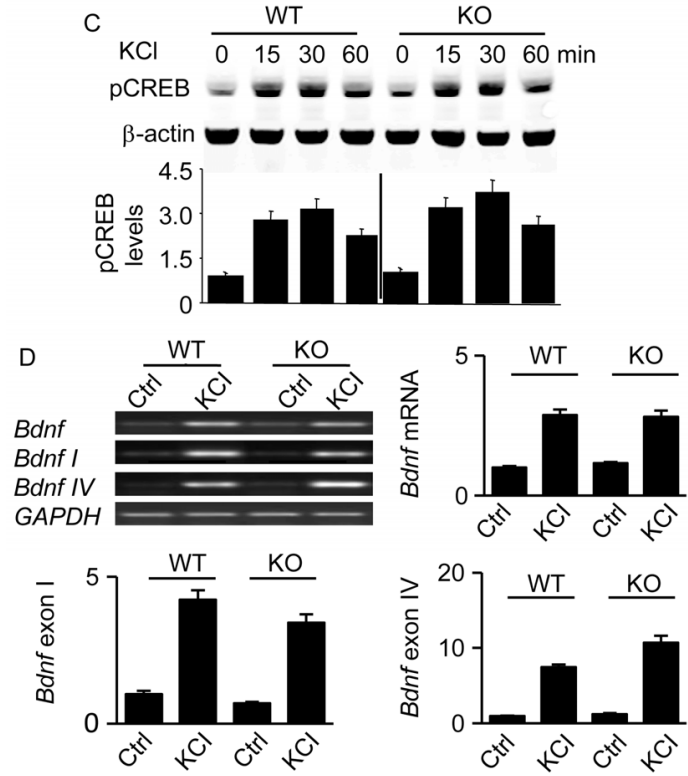

Figure $1 \mathrm{Ca}^{2+}$-stimulated up-regulation of CREB phosphorylation and $B d n f$ transcription in WT and CaMKIV KO neurons. A and C, The level of pCREB in control neurons and following the stimulation with NMDA (A) or $\mathrm{KCl}(\mathrm{C})$ was determined by Western blot. The relative level of pCREB was defined as one in the WT control neurons and normalized to the level of $\beta$-actin. Quantification was based on data collected from three separate experiments. Two-way ANOVA shows no significant difference between WT and CaMKIV KO neurons. B and D, The level of total, exon I-, and exon IV-containing $B d n f$ mRNA (as indicated) was determined in control and NMDA- (B) or KCl-stimulated (D) neurons by RT-PCR. The relative level of $B d n f$ mRNA in the WT control (Ctrl) group was defined as one and normalized to the level of $G A P D H$. Quantification was based on data collected from four separate experiments. Two-way ANOVA shows no significant difference between WT and CaMKIV KO neurons.

Thus, the basal locomotion and anxiety level in WT and CaMKIV KO mice responded equally to the contextual training device.
2.3 CaMKIV KO mice show normal passive avoidance memory but are defective in learning the TDPA task

We next used another paradigm to determine the function of CaMKIV in fear memory formation. During passive avoidance training, WT and CaMKIV KO animals showed similar crossover latency (Figure 4A). When tested $24 \mathrm{~h}$ later, both groups showed significant and comparable increase in crossover latency, indicating similar memory formation. We further examined mouse behavior in TDPA, during which the association between the darkness and the aversive electric foot shock was temporally dissociated. It took WT mice three training sessions to gradually establish memory and avoid entering the dark chamber (Figure 4B). However, there was no significant memory formation in CaMKIV KO mice, as indicated by no increase in crossover latency (Figure 4B).

\subsection{Function of CaMKIV in anxiety-related behavior and voluntary running activity}

One possibility that CaMKIV KO mice showed less crossover latency may be due to that they have more preference to the dark half of the passive avoidance chamber. Hence, we performed a light/dark test by using the same passive avoidance device. During the 10-min test, the two groups of mice showed similar latency for their first dark-to-light transition (Figure 5A). Interestingly, CaMKIV KO mice stayed in the lit chamber for longer time (Figure 5B). Both groups had similar number of transition between the lit and dark chambers (Figure 5C). These results indicate that CaMKIV KO mice are anxiolytic, and their shorter cross over latency during the TDPA training/testing is not due to that they tried to avoid light.

We further subjected WT and CaMKIV KO mice to marble-burying test, which measures repetitive behavior and anxiety level. Both groups buried similar number of marbles (Figure 6).

To determine the function of CaMKIV in motivation and motor function, we examined mouse behavior in voluntary wheel-running activity in its home cage for $7 \mathrm{~d}$. WT and CaMKIV KO animals showed similar daily running activity, as indicated by comparable running time (Figure 7A) and running distance (Figure 7B). CaMKIV KO mice showed significantly higher maximal running speed than WT animals (Figure 7C).

\section{Discussion}

Although it has been hypothesized that CaMKIV regulates CREB phosphorylation at Ser133, data from this and other studies demonstrated normal pCREB level in nonstimulated CaMKIV KO neurons (see Figure 1 and [11]). Intriguingly, we found that the up-regulation of pCREB 

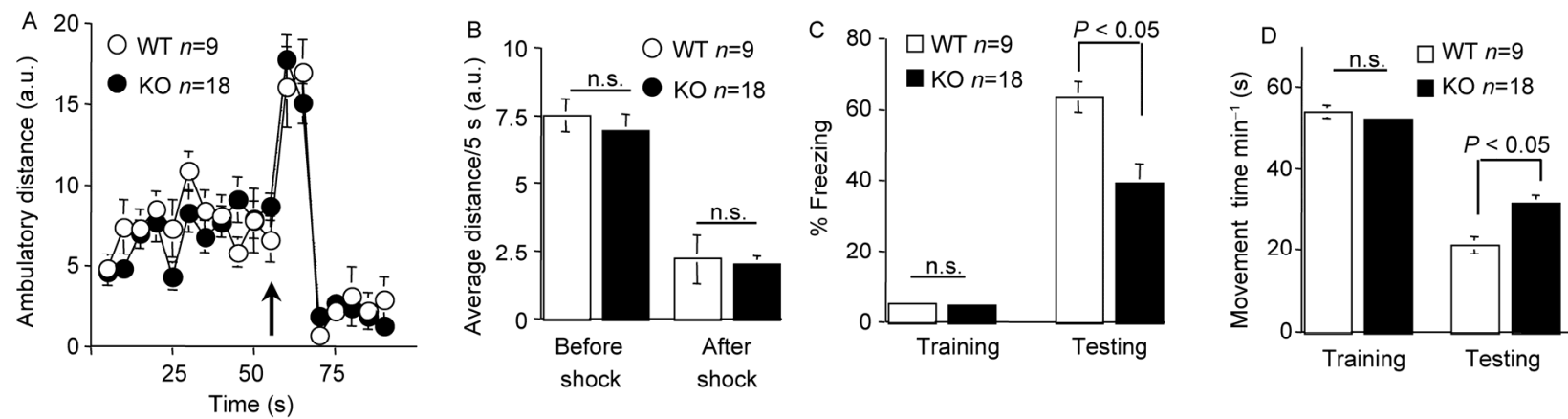

Figure 2 CaMKIV KO mice show impaired contextual fear memory. A, Ambulatory movement in each of the 5-s duration was recorded for WT and CaMKIV KO mice during contextual training. The arrow indicates the delivery of the mild foot shock. Repeated measures two-way ANOVA indicate no difference between WT and CaMKIV KO animals. B, Average movement before and after the mild foot shock during the training session are presented. C and D, Percentage of freezing (C) and average movement (D) during training (before the delivery of foot shock) and testing (24 h after training) are presented. n.s., not significant. a.u., arbitrary unit.
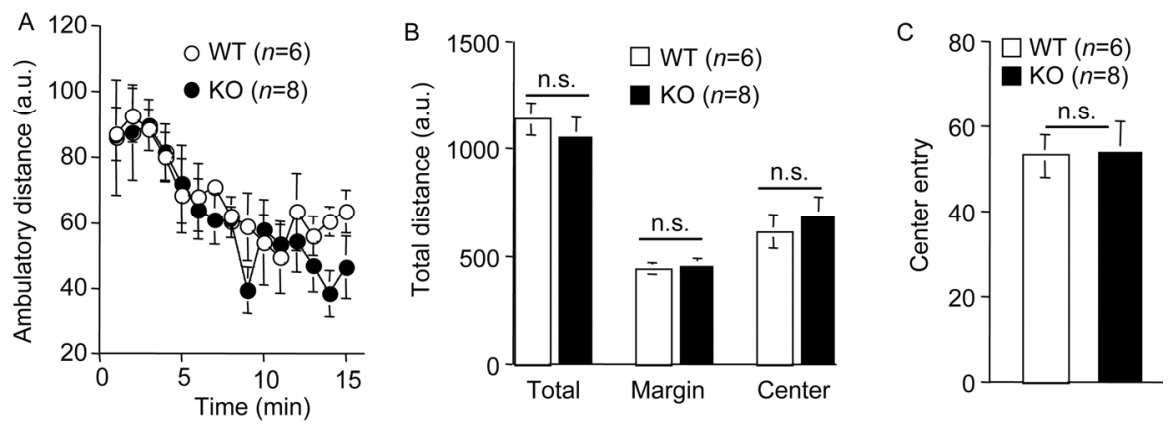

Figure 3 CaMKIV KO mice show normal locomotor activity and anxiety-related behavior in the open field test. Mouse was place to the chamber, which is also used for contextual training/testing, and allowed to move freely for $15 \mathrm{~min}$. A, Ambulatory movement for each of the 1-min bin is presented. Repeated measures two-way ANOVA indicates no difference between WT and CaMKIV KO animals. B, Total movement distance in the whole chamber, or the margin area, or the center area is presented. C, The number of entry to the center area of the chamber is presented. n.s., not significant. a.u., arbitrary unit.
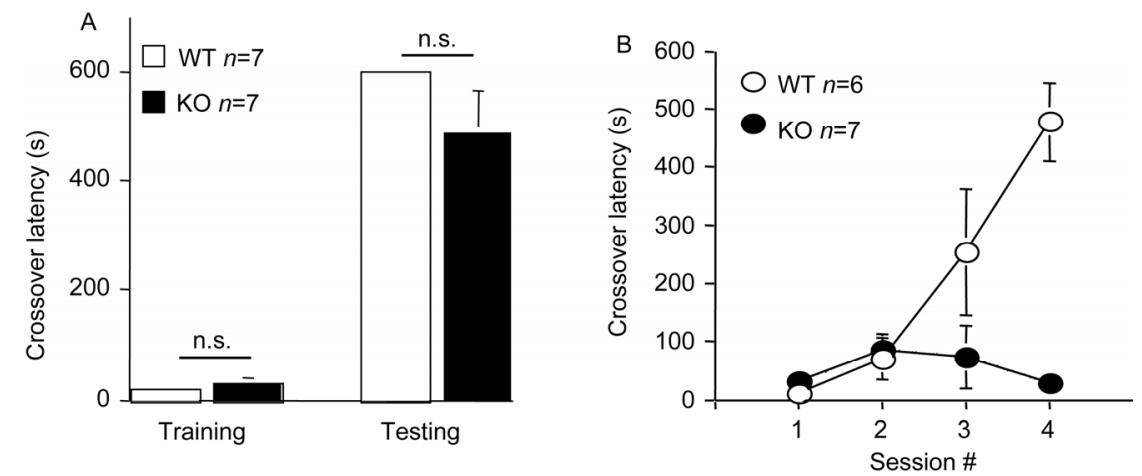

Figure 4 CaMKIV KO mice show normal passive avoidance memory but impaired TDPA performance. A, The crossover latency during passive avoidance training and testing is presented. n.s., not significant. B, The crossover latency during the four training/testing TDPA sessions is presented. Repeated measures two-way ANOVA indicate significant difference between WT and CaMKIV KO animals.

following NMDAR and L-VGCC activation does not require CaMKIV activity. The basal and $\mathrm{Ca}^{2+}$-stimulated transcription of the CREB target gene $B d n f$ was also comparable between WT and CaMKIV KO neurons. We postulate that CREB activity may be supported by other kinases such as ERK1/2 and cAMP-dependent protein kinase [21] in the absence of CaMKIV. Such possibility is supported by that learning-induced CREB phosphorylation [22] and Bdnf transcription [16] are impaired in mouse mutants of $\mathrm{Ca}^{2+}$-stimulated adenylyl cyclases. This is in contrast to the study by Ho et al. [10], which showed that the $\mathrm{KCl}$-induced pCREB is absent in CaMKIV KO neurons. The study further found that the glutamate-induced pCREB is also absent in the hippocampal slices of CaMKIV KO mice. It is evident that activity-dependent up-regulation of CREB phosphorylation in vivo requires CaMKIV activity. The increase 

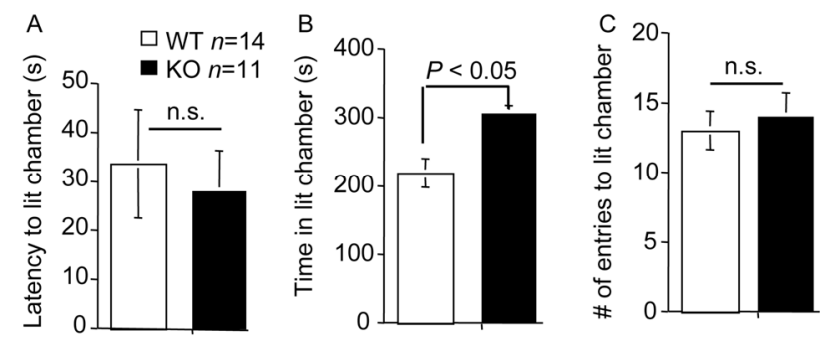

Figure 5 CaMKIV KO mice show less anxiety-like behavior in light/dark test. WT and CaMKIV KO mice were allowed to move freely for 10 min between the lit and dark chambers of the device, which is also used for passive avoidance. The crossover latency to the lit chamber (A), total time spent in the lit chamber (B), and the number of transition to the lit chamber (C) are presented. n.s., not significant.

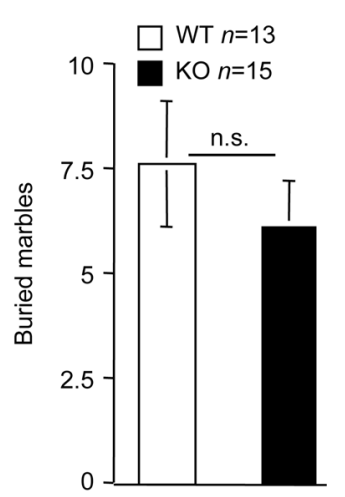

Figure 6 CaMKIV KO mice show normal behavior in the marble-burying test. The number of marbles buried during the 30-min test is presented. n.s., not significant.

in pCREB is impaired in CaMKIV KO mice [11] following fear conditioning. It is not clear whether the different outcome is due to the difference between synaptic activity and pharmacological manipulation, as well as the age of the neurons and genetic background.

In contrast to the lack of CaMKIV KO effects on $B d n f$ exon IV transcription, our previous study showed that overexpression of dominant-negative CaMKIV (dnCaMKIV) in cultured neurons partially suppressed the exon IV promoter activity following either $\mathrm{KCl}$ or NMDA stimulation [15].
Overexpression of dnCaMKIV also decreases the $\mathrm{Ca}^{2+}$ stimulated up-regulation of CRE activity [15]. It remains unclear whether dnCaMKIV also impinges on other intracellular signaling molecules other than the activity of CaMKIV. It is interesting to note that mice overexpression dnCaMKIV show different phenotypes when compared to CaMKIV KO mice. Impaired spatial memory was observed in dnCaMKIV animals [23] but not in CaMKIV KO mice [10].

The CaMKIV KO mice used in this study were backcrossed to the C57BL6 background from the strain, in which the exon III of the CaMKIV gene is deleted through homologous recombination [10]. Previous characterization shows that this specific KO strain (exon III KO) is defective in hippocampal long-term potentiation [10], a well-known cellular substrate of learning and memory. A subsequent study examined the hippocampus-dependent contextual fear memory, and found that the exon III KO mice fail to recall the conditioned contextual fear when tested 1 and $7 \mathrm{~d}$ but not $1 \mathrm{~h}$ after training [11]. This indicates that CaMKIV is required for memory retention rather than acquisition/ learning. The function of CaMKIV is further supported by that overexpression of CaMKIV in mice enhances contextual fear memory [24]. Surprisingly, a recent study using mice lacking exon I of CaMKIV (exon I KO) observed normal contextual memory in mutant animals when tested 1 and $7 \mathrm{~d}$ after training [13], considering that there is no detectable CaMKIV expression in either the exon III or the exon I KO mice. As an independent group, we confirmed that the exon III $\mathrm{KO}$ mice are defective in contextual memory retention but not acquisition (Figure 2). We further found that the locomotor activity and anxiety-like behavior in the setting same as the contextual training chamber are comparable between WT and the exon III KO mice. We noticed that, while the exon III KO mice received one electric foot shock (this study and [11]), the exon I KO mice received three shocks during the contextual training [13]. Thus, it is possible that the CaMKIV-regulated memory may be dependent on training intensity (i.e., one shock vs. three shocks). Secondly, Wiltgen et al. [25] have demonstrated that fear memory formed after receiving multiple
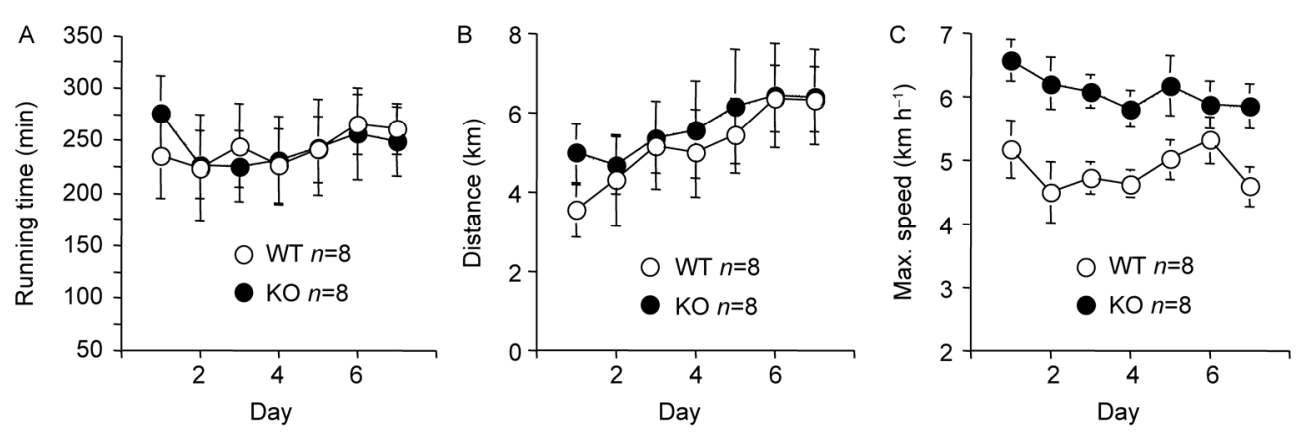

Figure 7 CaMKIV KO mice show higher maximal running speed during voluntary wheel-running in their home cage. Mouse had free access to a running wheel in its home cage. Daily wheel-running activity was recorded for $7 \mathrm{~d}$. A, Running time. B, Running distance. C, Maximal running speed. Repeated measures two-way ANOVA indicates significant difference between WT and CaMKIV KO animals for C but not for A and B. 
shocks does not require intact hippocampal function. In support of this idea, the exon I KO mice show defective contextual memory $28 \mathrm{~d}$ after training, when memory maintenance is mainly supported by cortical regions other than the hippocampus [26].

Our results along with the study by Takao et al. [13] demonstrate that passive avoidance memory does not require CaMKIV. However, when using TDPA as a modified passive avoidance test, CaMKIV KO mice did not show any measurable memory. Similar to passive avoidance, memory for TDPA also requires the function of hippocampus [27]. As the association of the conditioned stimulus (i.e. the dark chamber) with the aversive foot shock (i.e., the un-conditioned stimulus) is temporally delayed, we consider that learning the TDPA task is more demanding. The phenotype difference in passive avoidance and TDPA further suggests that the requirement of CaMKIV in learning and memory depends on training intensity.

Although CaMKIV KO mice did not show abnormal anxiety-related behavior in the contextual training chamber, they showed certain anxiolytic phenotype in the light/dark test. This is consistent with that the exon I and exon III KO mice show less anxiety-like behavior in the elevated plus maze $[12,13]$. Interestingly, previous studies fail to detect any major light/dark test phenotype in either exon I or exon III KO mice $[12,13]$. It is not clear whether all these studies have used equivalent lighting conditions.

The potential physiological function of CaMKIV in motor function is implicated by that CaMKIV KO mice show impaired cerebellum long-term depression [10]. However, when examined by the accelerating rotorod test, CaMKIV KO mice showed normal motor learning [10]. Both the exon I and exon III KO mice also display normal locomotion in the open filed test (this study and [13], but also see [28]). By examining the voluntary running activity in their home cages, we identified a new role of CaMKIV in regulating motor function, as indicated by higher maximal running speed in CaMKIV KO mice.

In summary, this study confirms that CaMKIV regulates contextual fear memory and certain aspect of anxietyrelated behavior. Our data also suggest new function of CaMKIV in passive avoidance memory and motor activity.

This work was supported by US National Institutes of Health grant (MH076906) to Wang HongBing.

1 Pivovarova NB, Andrews SB. Calcium-dependent mitochondrial function and dysfunction in neurons. Febs J, 2010, 277: 3622-3636

2 Grosskreutz J, Van Den Bosch L, Keller BU. Calcium dysregulation in amyotrophic lateral sclerosis. Cell Calcium, 2010, 47: 165-174

3 Wayman GA, Tokumitsu H, Davare MA, Soderling TR. Analysis of CaM-kinase signaling in cells. Cell Calcium, 2011, 50: 1-8

4 Redmond L, Kashani AH, Ghosh A. Calcium regulation of dendritic growth via CaM kinase IV and CREB-mediated transcription. Neuron, 2002, 34: 999-1010

5 Impey S, Fong AL, Wang Y, Cardinaux JR, Fass DM, Obrietan K,
Wayman GA, Storm DR, Soderling TR, Goodman RH. Phosphorylation of CBP mediates transcriptional activation by neural activity and CaM kinase IV. Neuron, 2002, 34: 235-244

6 Sun P, Enslen H, Myung PS, Maurer RA. Differential activation of CREB by $\mathrm{Ca}^{2+} /$ calmodulin-dependent protein kinases type II and type IV involves phosphorylation of a site that negatively regulates activity. Genes Dev, 1994, 8: 2527-2539

7 Chawla S, Hardingham GE, Quinn DR, Bading H. CBP: a signal-regulated transcriptional coactivator controlled by nuclear calcium and CaM kinase IV. Science, 1998, 281: 1505-1509

8 Sheng M, McFadden G, Greenberg ME. Membrane depolarization and calcium induce c-fos transcription via phosphorylation of transcription factor CREB. Neuron, 1990, 4: 571-582

9 Hall J, Thomas KL, Everitt BJ. Rapid and selective induction of BDNF expression in the hippocampus during contextual learning. Nat Neurosci, 2000, 3: 533-535

10 Ho N, Liauw JA, Blaeser F, Wei F, Hanissian S, Muglia LM, Wozniak DF, Nardi A, Arvin KL, Holtzman DM, Letoublon C, Bourgeon A, Delpero JR, Pezet D, Beaune P, Laurent-Puig P, Milano G.Linden DJ, Zhuo M, Muglia LJ, Chatila TA. Impaired synaptic plasticity and cAMP response element-binding protein activation in $\mathrm{Ca}^{2+}$ / calmodulin- depend- ent protein kinase type IV/Gr-deficient mice. J Neurosci, 2000, 20: 6459-6472

11 Wei F, Qiu CS, Liauw J, Robinson DA, Ho N, Chatila T, Zhuo M. Calcium calmodulin-dependent protein kinase IV is required for fear memory. Nat Neurosci, 2002, 5: 573-579

12 Shum FW, Ko SW, Lee YS, Kaang BK, Zhuo M. Genetic alteration of anxiety and stress-like behavior in mice lacking CaMKIV. Mol Pain, 2005, 1: 22

13 Takao K, Tanda K, Nakamura K, Kasahara J, Nakao K, Katsuki M, Nakanishi K, Yamasaki N, Toyama K, Adachi M, Umeda M, Araki T, Fukunaga K, Kondo H, Sakagami H, Miyakawa T. Comprehensive behavioral analysis of calcium/calmodulin-dependent protein kinase IV knockout mice. PLoS One, 2010, 5: e9460

14 Sekeres MJ, Neve RL, Frankland PW, Josselyn SA. Dorsal hippocampal CREB is both necessary and sufficient for spatial memory. Learn Mem, 2010, 17: 280-283

15 Zheng F, Zhou X, Luo Y, Xiao H, Wayman G, Wang H. Regulation of brain-derived neurotrophic factor exon IV transcription through calcium responsive elements in cortical neurons. PLoS One, 2011, 6: e28441

16 Zheng F, Zhou X, Moon C, Wang H. Regulation of brain-derived neurotrophic factor expression in neurons. Int $\mathrm{J}$ Physiol Pathophysiol Pharmacol, 2012, 4: 188-200

17 Thomas A, Burant A, Bui N, Graham D, Yuva-Paylor LA, Paylor R. Marble burying reflects a repetitive and perseverative behavior more than novelty-induced anxiety. Psychopharmacology (Berl), 2009, 204: 361-373

18 Tao X, Finkbeiner S, Arnold DB, Shaywitz AJ, Greenberg ME. Ca ${ }^{2+}$ influx regulates BDNF transcription by a CREB family transcription factor-dependent mechanism. Neuron, 1998, 20: 709-726

19 Hong EJ, McCord AE, Greenberg ME. A biological function for the neuronal activity-dependent component of Bdnf transcription in the development of cortical inhibition. Neuron, 2008, 60: 610-624

20 West AE, Chen WG, Dalva MB, Dolmetsch RE, Kornhauser JM, Shaywitz AJ, Takasu MA, Tao X, Greenberg ME. Calcium regulation of neuronal gene expression. Proc Natl Acad Sci USA, 2001, 98: 11024-11031

21 Wu GY, Deisseroth K, Tsien RW. Activity-dependent CREB phosphorylation: convergence of a fast, sensitive calmodulin kinase pathway and a slow, less sensitive mitogen-activated protein kinase pathway. Proc Natl Acad Sci USA, 2001, 98: 2808-2813

22 Sindreu CB, Scheiner ZS, Storm DR. $\mathrm{Ca}^{2+}$-stimulated adenylyl cyclases regulate ERK-dependent activation of MSK1 during fear conditioning. Neuron, 2007, 53: 79-89

23 Kang H, Sun LD, Atkins CM, Soderling TR, Wilson MA, Tonegawa $\mathrm{S}$. An important role of neural activity-dependent CaMKIV signaling 
in the consolidation of long-term memory. Cell, 2001, 106: 771-783

24 Fukushima H, Maeda R, Suzuki R, Suzuki A, Nomoto M, Toyoda H, Wu LJ, Xu H, Zhao MG, Ueda K, Kitamoto A, Mamiya N, Yoshida T, Homma S, Masushige S, Zhuo M, Kida S. Upregulation of calcium/calmodulin-dependent protein kinase IV improves memory formation and rescues memory loss with aging. J Neurosci, 2008, 28: 9910-9919

25 Wiltgen BJ, Sanders MJ, Anagnostaras SG, Sage JR, Fanselow MS. Context fear learning in the absence of the hippocampus. J Neurosci, 2006, 26: 5484-5491
26 Dudai Y. The neurobiology of consolidations, or, how stable is the engram? Annu Rev Psychol, 2004, 55: 51-86

27 Zhang M, Moon C, Chan GC, Yang L, Zheng F, Conti AC, Muglia L, Muglia LJ, Storm DR, Wang H. Ca-stimulated type 8 adenylyl cyclase is required for rapid acquisition of novel spatial information and for working/episodic-like memory. J Neurosci, 2008, 28: $4736-4744$

28 Ribar TJ, Rodriguiz RM, Khiroug L, Wetsel WC, Augustine GJ, Means AR. Cerebellar defects in $\mathrm{Ca}^{2+} / \mathrm{cal}_{\text {modulin kinase IV-deficient }}$ mice. J Neurosci, 2000, 20: RC107

Open Access This article is distributed under the terms of the Creative Commons Attribution License which permits any use, distribution, and reproduction in any medium, provided the original author(s) and source are credited. 